\title{
Maturation of Nephrons by Implanting hPSC- Derived Kidney Progenitors Under Kidney Capsules of Unilaterally Nephrectomized Mice
}

\section{Xin $\mathrm{Yu}$}

The second hospital,cheeloo college of medicine,shandong university

\section{Shan Jiang}

Shandong Provincial Hospital

\section{Kailin Li}

the second hospital,cheeloo college of medicine,shandong university

\section{Xianzhen Yang}

Shandong Provincial Hospital

\section{Zhihe Xu}

Shandong University

\section{Xiaohang Du}

Tianjin Baodi Hospital

\section{Feng Kong}

Shandong Provincial Hospital

Shengtian Zhao ( $\square$ zhaoshengtian@sdu.edu.cn )

the second hospital,cheeloo college of medicine, shandong university

\section{Research}

Keywords: kidney, organoids, induced pluripotent stem cells, implantation

Posted Date: September 17th, 2021

DOl: https://doi.org/10.21203/rs.3.rs-847264/v1

License: (1) (1) This work is licensed under a Creative Commons Attribution 4.0 International License. Read Full License 


\section{Abstract \\ Background}

Human pluripotent stem cell (hPSCs)-derived kidney organoids may contribute to disease modeling and generation of kidney replacement tissues. However, realization of such applications requires the induction of hPSCs into functional mature organoids. One of the key questions for this process is whether a specific vascular system exists for nephrogenesis. Our previous study showed that implantation of hPSCderived organoids below the kidney capsules of unilaterally nephrectomized immunodeficient mice for a short-term (2 weeks) resulted in the enlargement of organoids and production of vascular cells, although signs of maturation were lacking.

\section{Methods}

In this study, organoids are induced in vitro during 15 days and then sub-capsularly grafted into kidneys, we used the same unilaterally nephrectomized immunodeficient mice model to examine whether a medium -term (4 weeks) implantation could improve organoid maturation and vascularization, as evaluated by immunofluorescence and transmission electron microscopy(TEM).

\section{Results}

We demonstrate that after 2-4 weeks implantation, implanted renal organoids can form host-derived vascularization and mature in the absence of any exogenous vascular endothelial growth factor. Glomerular filtration barrier maturation was evidenced by glomerular basement membrane deposition, perforated glomerular endothelial cell development, as well as apical to basal podocyte polarization. A polarized monolayer epithelium and extensive brush border were also observed for tubular epithelial cells.

\section{Conclusions}

Our results indicate that the in vivo microenvironment is important for the maturation of human kidney organoids. Stromal expansion and a reduction of nephron structures were observed following longer-term (12 weeks) implantation,suggesting effects on off-target cells during the induction process. Accordingly, induction efficiency and transplantation models should be improved in the future.

\section{Introduction}

Globally, the annual number of individuals subjected to dialysis or kidney transplants for end-stage kidney disease (ESKD) is approximately 2.6 million. About 2.2 million patients with ESKD die because of therapeutic inaccessibility [1, 2]. Donor kidneys are insufficient in kidney transplantion and the survival expectancy of adults on long-term dialysis is barely a decade [3]. Therefore, it is urgent to prevent chronic 
renal disease development to ESKD. Accordingly, innovative models should be developed to improve the understanding of kidney disease pathogenesis and the potential of endogenous kidney regeneration.

Human pluripotent stem cells (hPSCs) provide a potential tool for therapy and investigation of disease pathophysiology. Takasato et al.[7] reported protocols for nephron formation from hPSCs involving generation of renal organoids in three-dimensional (3D) cultures by optimizing the exposure time, dose, and presence of key growth factors. Differentiation of these progenitor cells results in specialized nephron cell types, including podocytes, proximal tubule cells, thick ascending limb of the loop of Henle, and distal tubule cells [4-9]. However, a problem remains that the degree of maturation of these cells in vitro is generally low, as evidenced by inaccurate anatomical structures at either the transcriptional or morphological level [10]. This raises questions about whether maturity requires more time, alterations in culture conditions, and support from the blood supply.

Compensatory hypertrophy of the other kidney, which is a well-known clinical phenomena, has been shown to develop from unilateral nephrectomy $[11,12]$. The in vivo environment was shown to deliver nutrients to grafts of hPSC-derived kidney progenitors transplanted under renal capsules of unilaterally nephrectomized immunodeficient mice; however, shortterm (2 weeks) implantation did not enhance renal organ-related cell differentiation or maturation [13]. In this study, we aimed to determine whether longer implantation ( $>2$ weeks) of renal organoids placed renal subcapsular in unilaterally nephrectomized host immunodeficient mice could improve organoid maturation. The extent of glomerular, tubular, and vascular tissue maturation of grafts after 2, 4, and 12 weeks of transplantation was compared with that of organoids cultured in vitro. Our results suggest that prolonged (4 weeks) subcapsular implantation of renal organoids significantly improved glomerular, tubular, and vascular tissue maturation. However, increased interstitium and reduced nephron structures were observed 12 weeks after transplantation, suggesting that our induction method and transplantation model need further improvements.

\section{Materials And Methods}

Human induced pluripotent stem cells (hiPSCs). hiPSCs (Nuwacell ${ }^{\mathrm{TM}}$ ) were seeded in sixwell cell plates (Costar; Corning, Inc.; Cat. No. 3516). The plates were coated with Matrigel (Corning; Cat. No. 354277) and contained the mTeSR1 medium (Stem Cell Technologies, Inc.; Cat. No. 85850). Incubation was done at $37^{\circ} \mathrm{C}$ in a $5 \% \mathrm{CO}_{2}$ environment. Passaging of hiPSCs was done in an iPSCs dissociation solution (ReproCELL, Inc.; Cat. No. RCHETP002) at a ratio of 1:3 after every 7 days, as instructed by the manufacturer.

Induction of hPSCs to form kidney precursors in culture. When cells reached $50 \%$ confluence, Add Nephron differentiation medium sequentially as instructed by the manufacturer [13]. Organoids are induced in vitro during 15 days .On Day 15, kidney organoids were harvested for experiments [13].

Immunofluorescence analysis of $3 \mathrm{D}$ organoids. Frozen tissue sections stored at $-80^{\circ} \mathrm{C}$ were placed in cold methanol for 10 min, washed twice for 5 min each time using PBS and once using PBS $/ 0.3 \%$ Triton X-100 
for $5 \mathrm{~min}$. Then, they were incubated with $3 \%$ goat serum for $30 \mathrm{~min}$ at room temperatures. After draining the solution from tissue sections, overnight incubation of slides at $4^{\circ} \mathrm{C}$ was done with indicated primary antibodies (Abcam) and dilutions: antiWT1 (1:50; Cat. No. ab89901), antiECAD (1:100; Cat. No. ab1416), antiPODXL (1:100; Cat. No. ab150358), anti-MECA-32 (1:200, Cat. No. ab27853), antiCD31 (1:200; Cat. No. ab24590), anti-human nuclear antigen (HNA; 1:1000; Cat. No. ab191181), and anti-Nanog (1:100; Cat. No. ab109250). Then,slides were washed thrice (for $1 \mathrm{~h}$ each time) using PBS. The fourth wash was done at $4^{\circ} \mathrm{C}$ overnight. Biotinylated Lotus tetragonolobus lectin (LTL; 1:300; Cat. No. B1325; Vector Laboratories, Inc.) immunostaining was performed using a Streptavidin/Biotin Blocking Kit (Cat. No. SP2002; Vector Laboratories) as instructed by the manufacturer. Organoid sections were incubated for 1 $\mathrm{h}$ at room temperature in the presence of secondary antibodies [1:500; Alexa Flour 488-labeled goatantimouse IgG (Cat. No. A0428) or Cy3labeled goatantirabbit IgG (Cat. No. A0516), Beyotime Institute of Biotechnology; or mlgGk BPPE (Cat. No. SC516141), Santa Cruz Biotechnology, Inc.] in an antibody dilution buffer. Then, they were washed thrice (30 min each time) using PBS after which nuclear staining was performed with 6-diamidino-2-phenylindole. Finally, sections were mounted using Vectashield (Vector Laboratories; Cat. No. H1200) and evaluated by confocal microscopy at $\times 200$ and ×400 (Zeiss AG; Model No. LSM780).

Animal surgery. The care and handling of animals was done as instructed by the the National Institutes of Health Guide for the Care and Use of Laboratory Animals. The Ethics Committee of The Second Hospital of Shandong University approved this study. Twenty male immunodeficient mice (NOD SCID; aged 5-6 weeks; weighing 22-25 g; from Beijing Vital River Laboratory Animal Technology Company) were anesthetized with pentobarbital sodium $(1 \% ; 50 \mathrm{mg} / \mathrm{kg})$ through intraperitoneal administration. Right mice kidneys were ligated and removed. Then, the left kidney side of the back was opened. After kidney exposure, the renal capsule was sliced to establish a small mouth. The D15 kidney organoids were administered via the opening of the receptor kidney. We implanted organoids in 3 groups of mice, some differentiated for 2 weeks $(n=15)$ and 4 weeks $(n=15)$, and others for 12 weeks $(n=20)$. Mice were sacrificed at 2, 4, and 12 weeks post-injection. After the experiment, mice euthanasia was done through intraperitoneal administration of pentobarbital sodium $(1 \% ; 150 \mathrm{mg} / \mathrm{kg})$.

Hematoxylin and eosin staining (H\&E). Fixing of the graft was done for $48 \mathrm{~h}$ in paraformaldehyde (4\%) at room temperature. Then, the graft was embedded in paraffin and sliced into $4-\mu \mathrm{m}$-thick sections. A H\&E staining kit (Cat. No. G1120; Beijing Solarbio Science \& Technology Co, Ltd.) and Masson's trichrome staining kit (Cat. No. G1340; Beijing Solarbio Science \& Technology) were used as instructed by the manufacturers. Imaging was done by light microscopy at ×100.

Transmission electron microscopy (TEM). Renal organoids were chipped into 1-mm ${ }^{3}$ tissue blocks on ice and immediately put into $3 \%$ glutaraldehyde in a cacodylate buffer for $2 \mathrm{~h}$ at $4^{\circ} \mathrm{C}$. Then, organoids were post-fixed in a osmium-tetroxide phosphate buffer solution ( $1 \%)$ for $2 \mathrm{~h}$, dehydrated in a series of graded ethanol with acetone, permeated, and embedded in an epoxide resin. Semi-thin section localization was used to generate ultrathin slices $(70 \mathrm{~nm})$, which were stained by lead citrate and uranyl acetate. Sections were evaluated by JEM-1200EX TEM using a Marada-G2 type CCD photo ultrastructure for imaging. 


\section{Results}

\section{Organized glomerular and tubular structures observed by immunofluorescence}

When hiPSCs were seeded in a 6-well cell plate $\left(2,0000\right.$ cells per $\left.\mathrm{cm}^{2}\right)$ containing the mTeSR 1 medium, they were induced into renal organoids cells through a renal epithelial cell differentiation kit(Figure 1A). On Day 15 , kidney organoids were typically $3-5 \mathrm{~mm}$ in diameter and a nephron had formed within the organoids (FigureB). H\&E staining revealed that, in vitro, hiPSCs were prompted into forming a tubular structure (Figure1B). The formation of glomerular structures was evidenced by the presence of podocytes, as identified by PODXL and WT1 expression. Renal tubule structures including proximal tubules (LTL+) as well as distal tubules ( $\mathrm{CDH} 1+)$ were also observed (Figure1C).

To assess glomerular and tubular structure organization and maturation, TEM analysis of the organoids on the $15^{\text {th }}$ day was performed. TEM confirmed the presence of podocyte cells in organoids, as characterized by their well-developed cytoskeleton in the cytoplasmic matrix (Figure 2A-a), abundant cellular protrusions (Figure 2A-b), uneven nuclear membrane (Figure 2A-c), and epithelioid junction structures (Figure 2A-d); however, no capillaries were found around podocytes. Compared with human mature podocytes in vivo, podocytes in organoids cultured in vitro had short and irregular foot processes, and fewer organelles such as mitochondria and endoplasmic reticulum [14].

Renal tubules with tubular structures were also observed, including many rod-shaped mitochondria within cells, intracellular pleats at the base of cells (Figure 2B-e), and microvilli in the cell lumen (Figure 2B-f). Some tubules in vitro were multilayered (Figure 2B-h). However, the lumen was absent or small, the microvilli were short and disorderly (Figure 2B-f), and the nucleo-cytoplasmic ratio was large (Figure 2Be). These observations indicate that the epithelial cells were in an unstable immature stage. With the extension of in vitro culture, epithelial cells exhibited apoptosis (Figure 2B-g).

\section{Upon in vivo transplantation, Kidney organoids were vascularized}

Mouse metanephric tissue transplantation was performed as previously described $[15,16]$. Upon implantation under the renal capsule of recipient immune-compromised single-nephrectomized mice, organoid maturation degree was evaluated. Then, day 15 kidney organoids were implanted into renal capsules of unilateral-nephrectomized mice for up to 4 weeks. With increased time of implantation, the organoids grew progressively (Figure 3A), red blood cells became more abundant in glomeruli and grafts, and tubules formed a larger lumen; simultaneously, increased cartilaginous nonrenal tissue was observed (Figure 3B).

Immunofluorescence results demonstrated the existence of glomerular as well as tubular structures within organoids after implantation (Figure 3C). Macroscopically, the implanted tissues were invaginated by branched vessels that originated from host kidney regions (Figure 3A-c,d). Histologic analysis showed several maturing glomeruli with glomerular arterioles (Figure 3B). Red blood cells were found in maturing tissues of vascular as well as glomerular structures (Figure 3B), implying a connection with the recipients 
vascular system. Positive expression of HNA in the graft indicated that graft cells originated from human cells (Figure 3C). Mouse endothelial cell antigen-32 (MECA-32) staining revealed that positive cells were diffusely distributed in the glomerular tufts of implanted tissue (Figure 3C).

Kidney organoid implants were composed of segregated kidney structures with tubules, glomeruli and cartilage islands (Figure3B,C). Additionally, positive expression of NANOG in cartilage indicated that the graft retained differentiation potential (Figure 3C). In the 2 weeks implantation group,11/15 implants formed glomeruli,tubules and cartilage tissues. In the 4 weeks implantation group, 9/15 implants formed glomeruli, tubules and cartilage tissues.

\section{Ultrastructural examination of transplanted organoids showed graft vascularization as well as maturation in vivo}

After 4 weeks of implantation, podocytes had spread along basement membranes and formed basal adherens junctions near a clear trilaminar glomerular basement membrane (GBM), implying progression to slit diaphragm formation between the podocyte foot processes (Figure 4A-a,b,c). However, such an organized GBM was lacking in organoids cultured in vitro (Figure 2A). TEM also showed that the glomeruli in implants exhibited a characteristic podocyte arrangement on the outer blood capillary surfaces (Figure 4A-a,c). The capillaries had red blood cells, further implying that the glomeruli had a connection with host blood supply. TEM imaging of podocyte-capillary interfaces showed that some exhibited ultrastructures that were indistinguishable from those of mature glomeruli (Figure $4 \mathrm{~A}-\mathrm{a}, \mathrm{c})$. The podocytes exhibited a characteristic foot process joined by slit diaphragm-like structures, and underneath, urinary spaces were visible (Figure 4A-a,c). A fused GBM was also present (Figure 4A-a,b,c). Evidence of post-transplantation organoid maturation is encouraging, even when compared to the GBM, the fenestrated endothelium, and to mature podocyte structures of adult kidneys [14].

Within implanted grafts, tubular structures showed progressive maturation after 4 weeks of implantation, leading to epithelial polarization into a single monolayer as well as formation of well-developed apical brush border regions (Figure 4B-d,e). Moreover, tubular lumina progressively widened and peritubular capillaries aligned the tubules (Figure 4B-d,f), as observed in normal human kidneys. Additionally, signs of apicobasal polarization could be observed, including basally located nuclei and microvilli located on the luminal surface of cells (Figure 4B-d,e).

\section{Structural degeneration of nephrons and loss of cartilage at long-term follow-up}

Implanted kidney organoids survived until more than 12 weeks after transplantation. After 12 weeks, graft size continued to increase(Figure 5A), although nephron-like structures appeared to be degenerated; indeed, well-organized nephron-like structures were rarely observed at 12 weeks after implantation (Figure 5B,C). Moreover, glomerular structures almost completely disappeared and only a few tubules were observed (Figure 5C). 
Cartilage formation was observed in implanted kidney organoid grafts at 2 weeks after implantation

(Figure 3B). HNA was expressed in the observed cartilaginous masses in implanted kidney organoids (Figure 3C). Amounts of cartilage were increased at 4 weeks compared with 2 weeks after transplantation. However, after 12 weeks of transplantation, cartilage structures had disappeared and many mesenchymal tissues appeared (Figure 5B). In the 12 weeks group implants, mesenchymal tissues was observed in 14/20 implants.

\section{Discussion}

Stem cell-derived organoids have been a major advance for in vitro modeling of development and disease in recent years $[6,7,17,18]$. Because of their complex 3D structures, which may closely resemble in vivo tissue, organoids provide an advantage over monolayer culture [7]. However, the degree of maturation achieved in vitro is generally low, which remains a challenge for the field. In our previous study, volumes of renal organoids significantly increased after 2 weeks of implantation under renal capsules of unilaterally nephrectomized mice compared with identical organoids cultured in vitro. The present study provides evidence that longer ( $>2$ weeks) implantation not only enhanced the volume of organoids, but improved their maturation.

Numerous studies have shown that after unilateral nephrectomy, compensatory hypertrophy as well as hyperfunctions of nephrons occur in the remaining kidney as a result of inductions of various growth factors [19]. Matsumoto et al.[20] reported that grafts become well differentiated after fetal kidney transplantation into omenta or abdominal aortas of unilaterally nephrectomized rats. A related mechanism may be associated with enhanced transplanted renal organoid development in unilaterally nephrectomized recipients observed in this and our previous study [13]. In the present study, maturation of kidney organoids was evidenced by GBM, mature podocyte structures, and well-developed apical brush borders. Furthermore, 4 weeks of implantation promoted glomerular filtration barrier maturation accompanied by GBM deposition, fenestrated glomerular endothelium development, and apical-basal podocyte polarization. Moreover, maturation of tubular epithelia was associated with polarized singlelayer epithelium development and extensive brush borders in proximal tubules, which can be observed in the mature kidney structure [21]. However, time-matched non-transplanted organoids did not display any of these characteristics, instead remaining immature structurally and disorganized.

The invasion of endothelial cells into the glomerulus to form capillary loops is a key event occurring in the glomerulus and a prerequisite for filtration of the blood supply. Previous experiments, in which human fetal kidney tissues were implanted under the kidney capsule, confirmed the capacity of a PSC-derived model to regenerate human kidney tissues [22]. Moreover, implant-generate glomeruli had GBM and endothelial cells, providing capillary loops with mechanical integrity in vivo. Moreover, the lack of requirement for VEGF may be advantageous for future therapeutic applications of PSC kidney progenitors. We also demonstrated that after subcapsular implantation of hPSC-derived renal precursors, the glomeruli had significant capillaries, some of which contained red blood cells. As both MECA-32+ cells and CD31 + cells were dispersed in glomerular clusters in the transplanted tissue, we speculate that 
the vascular network could originate from hPSCs and contribute to graft vascularization within organoids through anastomosis to endothelial plexus from hosts, which recapitulates kidney vasculature embryonic development, whereby angiogenic hemangioblast precursor cells and vasculogenic endothelial precursors within organs are needed for glomerular microcirculation development [23].

While it is difficult to evaluate gestational age from the morphology of an individual nephron, prolonged transplantation may further mature the induced nephron [23]. A vascular network was existent in kidney organoids at 2 weeks transplantation (Fig. 3A and 3B). Moreover, upon implantation, a part of this network became connected to host circulation. The absence of pre-existing vascular network ingrowth into the glomeruli of cultured organoids in vitro reflects less permissive culture conditions for vasculogenic activities within organoids. Compared with short-term implantation ( $<2$ weeks), prolonged transplantation (2-4 weeks) could increase the amount of red blood cells in organoids and extent of maturity of nephron structures. This may be attributed to increased angiogenesis in organoids, which can thus obtain more host-derived nutrients.

However, we observed excessive growth of mesenchymal tissue at 12 weeks after transplantation, presumably caused by nonrenal cells in transplants. This observation suggests the differentiation potential of organoids extends beyond renal cells. The phenomenon of nephron structure and cartilage loss after long-term transplantation suggests that the initially transplanted cells included non-kidney or partially differentiated cells; moreover, the renal transplantation microenvironment cannot completely replicate the embryonic kidney development microenvironment.

There remain limitations to overcome before hPSCs can produce a fully functional kidney. First, tissue from hPSC-derived renal precursors currently lacks arterial blood supply. Second, no structures resembling the renal pelvis or ureter have been found in transplanted organoids. Third, chondroid structures were observed in transplanted renal organoids, suggesting off-target effects on cells during the induction process. Thus, the induction efficiency should be improved in the future.

\section{Conclusions}

We obtained more mature kidney structures by implanting hPSC-derived kidney progenitor cells for 2-4 weeks under kidney capsules in unilaterally nephrectomized mice. Compared with non-transplanted organoids, transplanted organoids exhibited more mature glomeruli and tubules as well as nephron vascularization. More mature and vascularized renal organoids may offer new possible solutions to the shortage of kidney supply in kidney transplantation.

\section{List Of Abbreviations}




\begin{tabular}{|ll|}
\hline hPSCs & Human pluripotent stem cell \\
\hline TEM & transmission electron microscopy \\
\hline ESKD & end-stage kidney disease \\
\hline 3D & three-dimensional \\
\hline H\&E & Hematoxylin and eosin staining \\
\hline GBM & glomerular basement membrane \\
\hline
\end{tabular}

\section{Declarations}

\section{Acknowledgments}

The authors would like to thank Dr Xiulin Zhang and Dr Yong Guan(Department of Urology,The Second Hospital,Cheeloo College of Medicine,Shandong University

And Department of Urology, Shandong Provincial Hospital) for providing helpful discussions and technical support concerning the present study. In addition,the authors also thank Professor Boqin Li $\triangle$ Shandong WeiYa Biotechnology Co. ,Ltd囚for guidance on the implementation and interpretation of TEM.

\section{Ethics approval}

Animal care and handling follow the The National Institutes of Health Guide for the Care and Use of Laboratory Animals.

\section{Consent to participate}

All procedures were approved by The Ethics Committee of The Second Hospital,Cheeloo College of Medicine,Shandong University

\section{Consent for publication}

Not applicable.

\section{Authors' contributions}

$X D$ and FK conducted the study. SZ and $X Y$ designed the experiments. SJ, KL,XY and ZX performed experiments. All authors have read and approved the manuscript.

\section{Funding}

The present study was supported by the National Natural Science Foundation of China (grant no. 81670625,81970578 and 82074171) and"Shandong Provincial Hospital Affiliated to Shandong First Medical University"academic promotion programme (grant no.2020LI001). 


\section{Competing interests}

All authors claim that they have no competing interests.

\section{Availability of data and materials}

The datasets used in this paper are available on reasonable request from corresponding author.

\section{References}

1. Knowles SR, Ski CF, Langham R, O'Flaherty E, Thompson DR, Rossell SL, Moore G, Hsueh YS, Castle DJ. Design and protocol for the Dialysis Optimal Health Program (DOHP) randomised controlled trial. Trials. 2016;17:447.

2. Tandukar S, Hou S, Yabes J, Ran X, Fletcher M, Strollo P, Patel SR, Unruh M, Jhamb M. Does Kidney Transplantation Affect Sleep and Fatigue in Patients With Kidney. Disease? Transplant Direct. 2019;5:e461.

3. Vanholder R, Van Laecke S, Glorieux G, Verbeke F, Castillo-Rodriguez E, Ortiz A. (2018). Deleting Death and Dialysis: Conservative Care of Cardio-Vascular Risk and Kidney Function Loss in Chronic Kidney Disease (CKD). Toxins (Basel) 10.

4. Takasato M, Little MH. A strategy for generating kidney organoids: Recapitulating the development in human pluripotent stem cells. Dev Biol. 2016;420:210-20.

5. Morizane R, Bonventre JV. Generation of nephron progenitor cells and kidney organoids from human pluripotent stem cells. Nat Protoc. 2017;12:195-207.

6. Grassi L, Alfonsi R, Francescangeli F, Signore M, De Angelis ML, Addario A, Costantini M, Flex E, Ciolfi A, Pizzi S, Bruselles A, Pallocca M, Simone G, Haoui M, Falchi M, Milella M, Sentinelli S, Matteo PD, Stellacci E, Gallucci M, Muto G, Tartaglia M, De Maria R. and D Bonci. (2019). Organoids as a new model for improving regenerative medicine and cancer personalized therapy in renal diseases. Cell Death Dis 10.

7. Kaushik G, Ponnusamy MP, Batra SK. Current Status of 3-D Organoids as Pre-Clinical Models. Stem Cells. 2018;36:1329-40.

8. Lam AQ, Freedman BS, Bonventre JV. Directed differentiation of pluripotent stem cells to kidney cells. Semin Nephrol. 2014;34:445-61.

9. Yamaguchi S, Morizane R, Homma K, Monkawa T, Suzuki S, Fujii S, Koda M, Hiratsuka K, Yamashita M, Yoshida T, Wakino S, Hayashi K, Sasaki J, Hori S, Itoh H. Generation of kidney tubular organoids from human pluripotent stem cells. Sci Rep. 2016;6:38353.

10. van den Berg CW, Ritsma L, Avramut MC, Wiersma LE, van den Berg BM, Leuning DG, Lievers E, Koning M, Vanslambrouck JM, Koster AJ, Howden SE, Takasato M, Little MH. TJ Rabelink. (2018). Renal Subcapsular Transplantation of PSC-Derived Kidney Organoids Induces Neo-vasculogenesis and Significant Glomerular and Tubular Maturation In Vivo. Stem Cell Reports 10:751-65. 
11. Urie BK, Tillson DM, Smith CM, Brawner WR, Almond GT, Beard DM, Lenz SD, Lothrop CD Jr. Evaluation of clinical status, renal function, and hematopoietic variables after unilateral nephrectomy in canine kidney donors. J Am Vet Med Assoc. 2007;230:1653-6.

12. Kendi Celebi Z, Peker A, Kutlay S, Kocak S, Tuzuner A, Erturk S, Keven K, Sengul S. Effect of unilateral nephrectomy on urinary angiotensinogen levels in living kidney donors: 1 year follow-up study. $J$ Renin Angiotensin Aldosterone Syst. 2017;18:1470320317734082.

13. Zhang D, Du X, Zhang X, Li K, Kong F, Cheng G, Zhao S. In vitro induction and in vivo engraftment of kidney organoids derived from human pluripotent stem cells. Exp Ther Med. 2020;20:1307-14.

14. Hyodo T, Naguro T, Kameie T, lino A, Miyagawa I. Scanning and transmission electron-microscopic study of the development of the podocyte in the human fetus. Pediatr Nephrol. 1997;11:133-9.

15. Dekel B, Shezen E, Even-Tov-Friedman S, Katchman H, Margalit R, Nagler A, Reisner Y. Transplantation of human hematopoietic stem cells into ischemic and growing kidneys suggests a role in vasculogenesis but not tubulogenesis. Stem Cells. 2006;24:1185-93.

16. Hammerman MR. Pancreas and kidney transplantation using embryonic donor organs. Organogenesis. 2004;1:3-13.

17. Combes AN, Zappia L, Er PX, Oshlack A, Little MH. (2019). Single-cell analysis reveals congruence between kidney organoids and human fetal kidney. Genome Med 11.

18. Przepiorski A, Sander V, Tran T, Hollywood JA, Sorrenson B, Shih JH, Wolvetang EJ, McMahon AP, Holm TM. AJ Davidson. (2018). A Simple Bioreactor-Based Method to Generate Kidney Organoids from Pluripotent Stem Cells. Stem Cell Reports 11:470-84.

19. (2017). The Impact of Kidney Development on the Life Course: A Consensus Document for Action. Nephron 136:3-49.

20. Matsumoto K, Yokoo T, Yokote S, Utsunomiya Y, Ohashi T, Hosoya T. Functional development of a transplanted embryonic kidney: effect of transplantation site. J Nephrol. 2012;25:50-5.

21. Roy A, Al-bataineh MM, Pastor-Soler NM. Collecting duct intercalated cell function and regulation. Clin J Am Soc Nephrol. 2015;10:305-24.

22. Takasato M, Little MH. The origin of the mammalian kidney: implications for recreating the kidney in vitro. Development. 2015;142:1937-47.

23. Sharmin S, Taguchi A, Kaku Y, Yoshimura Y, Ohmori T, Sakuma T, Mukoyama M, Yamamoto T, Kurihara H, Nishinakamura R. Human Induced Pluripotent Stem Cell-Derived Podocytes Mature into Vascularized Glomeruli upon Experimental Transplantation. J Am Soc Nephrol. 2016;27:1778-91.

\section{Figures}




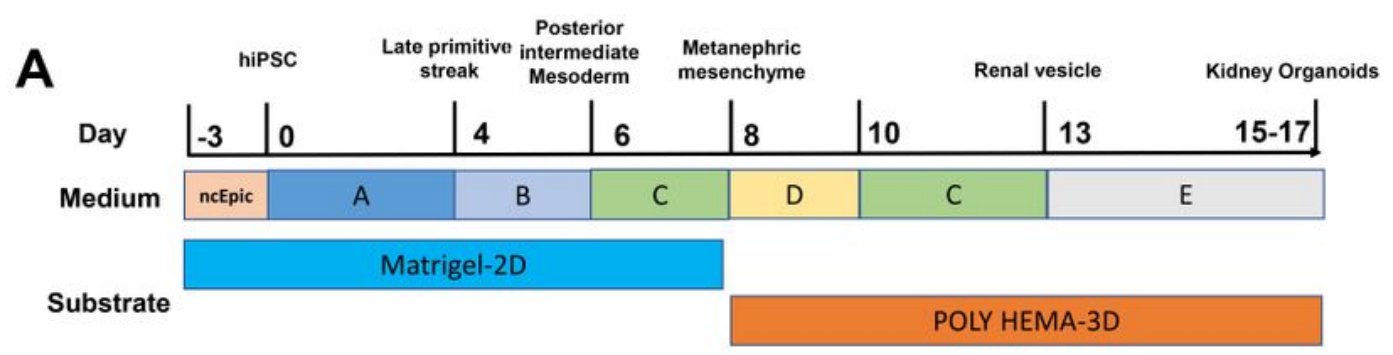
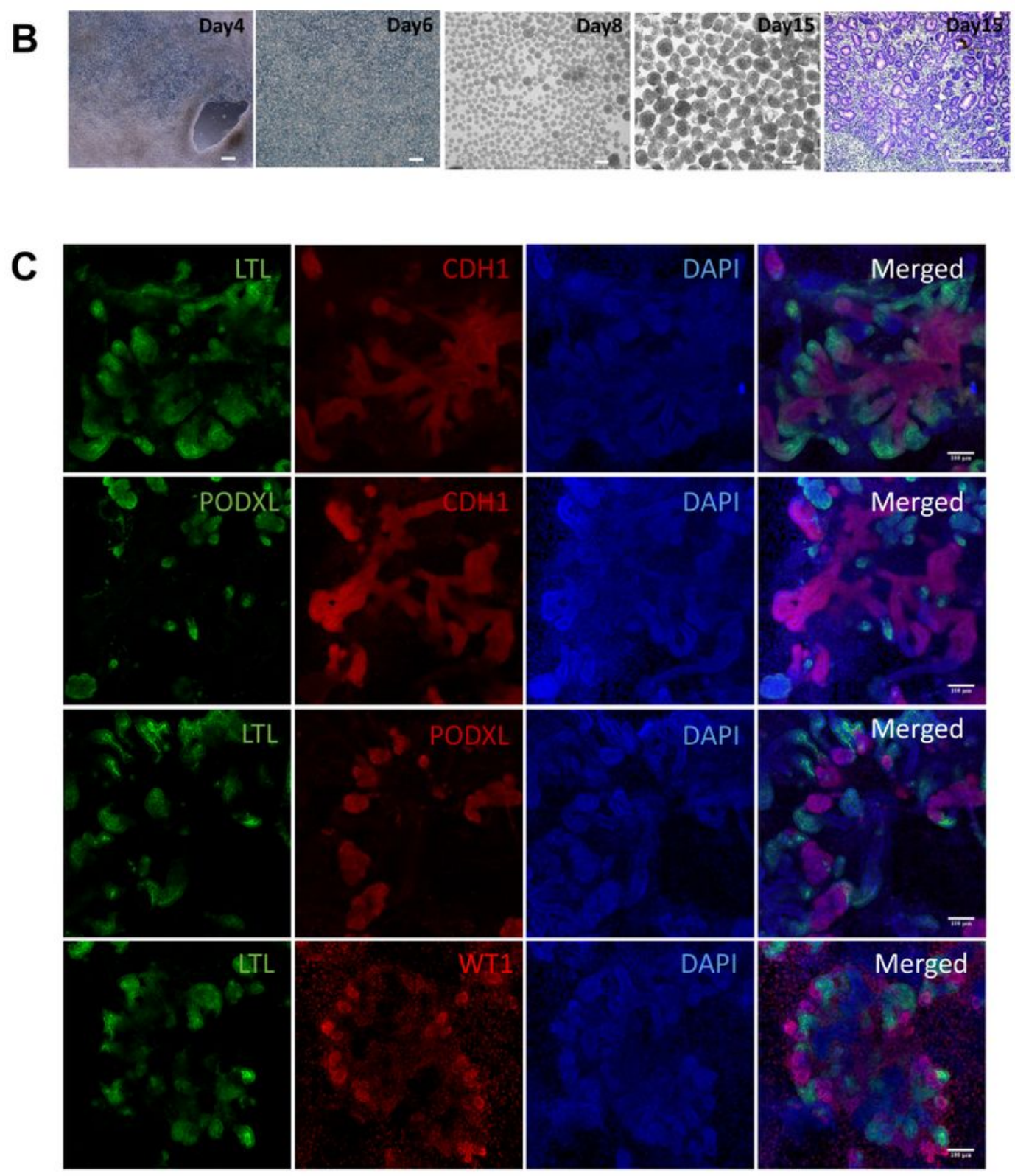

\section{Figure 1}

Human induced pluripotent stem cell (hiPSCs)-derived kidney organoids exhibited structural characteristics of a nephron on day 15 of differentiation. (A) Schematic of directed differentiation protocol to generate human renal organoids from hiPSCs. (B) Representative photos at the indicated differentiation stages and H\&E staining display structures of tubular in organoid after 15 days of differentiation. Scale bar, 50 m. (C) Immunofluorescence analysis of different nephron segments: 
podocytes (PODXL+هWT1+), proximal tubule $(\mathrm{LTL}+)$, distal tubule $(\mathrm{CDH} 1+)$. The tubular structure of proximal tubules (LTL+) and distal tubules $(\mathrm{CDH} 1+)$ adjoin each other, while podocytes (PODXL+/WT1+) are localized primarily at the end of the tubular structure (LTL+). Scale bar, $100 \mu \mathrm{m}$.

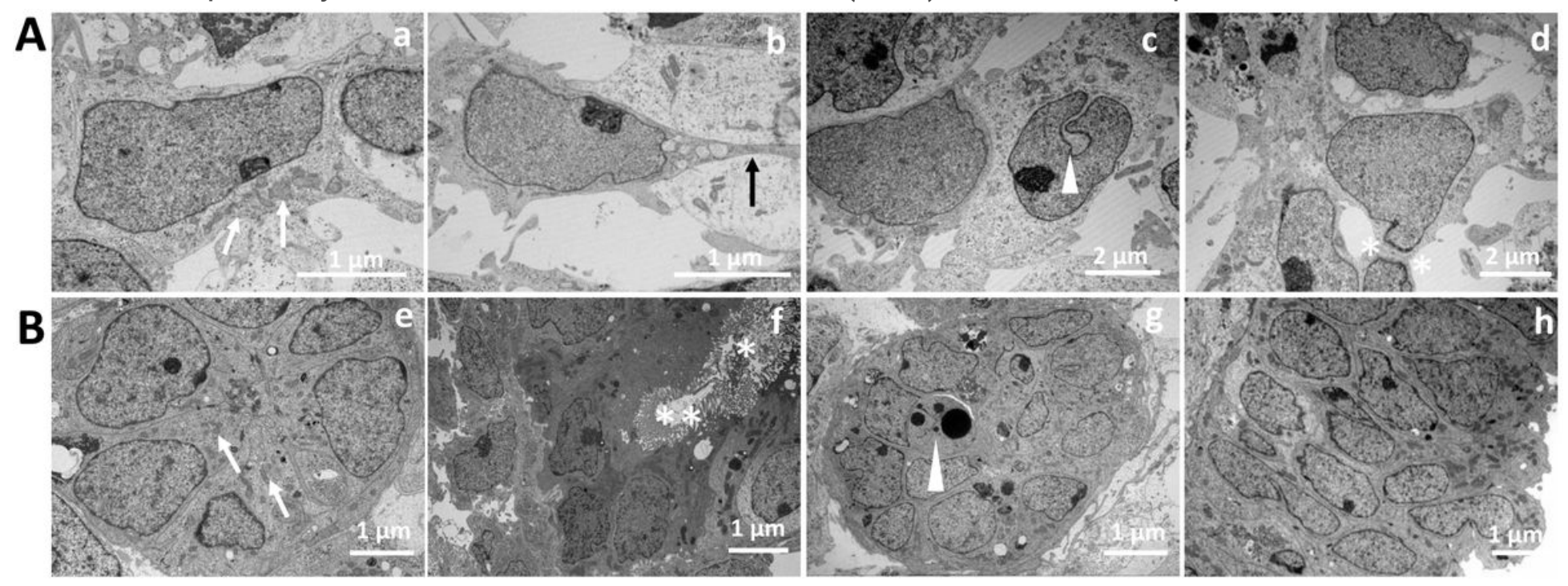

Figure 2

Ultrastructural evaluation revealed immaturity of kidney organoids cultured in vitro for 15 days. (A) Highmagnification transmission electron microscopy (TEM) images demonstrate characteristic podocyte structures. a, Cells containing a large number of organelles (white arrows); $b$, cells protrude to display a primitive foot (black arrow); c, uneven nuclear membrane structure of the cell (white arrowhead); $d$, epithelial junctions between cells (white asterisks). Scale bar, $1 \mu \mathrm{m}$. (B) TEM tile scan of kidney organoids cultured for 15 days displaying tubular structures. e, Overview TEM image of a cross-section of a tubule and numerous mitochondria (white arrows); $f$, brush border with microvilli (white asterisks) in the open lumen of a tubular structure; $\mathrm{g}$, apoptosis characterized by nuclear fragmentation (white arrowhead); $\mathrm{h}, \mathrm{a}$ portion of epithelial cells showed a layered epithelial structure, Scale bar, $1 \mu \mathrm{m}$. 


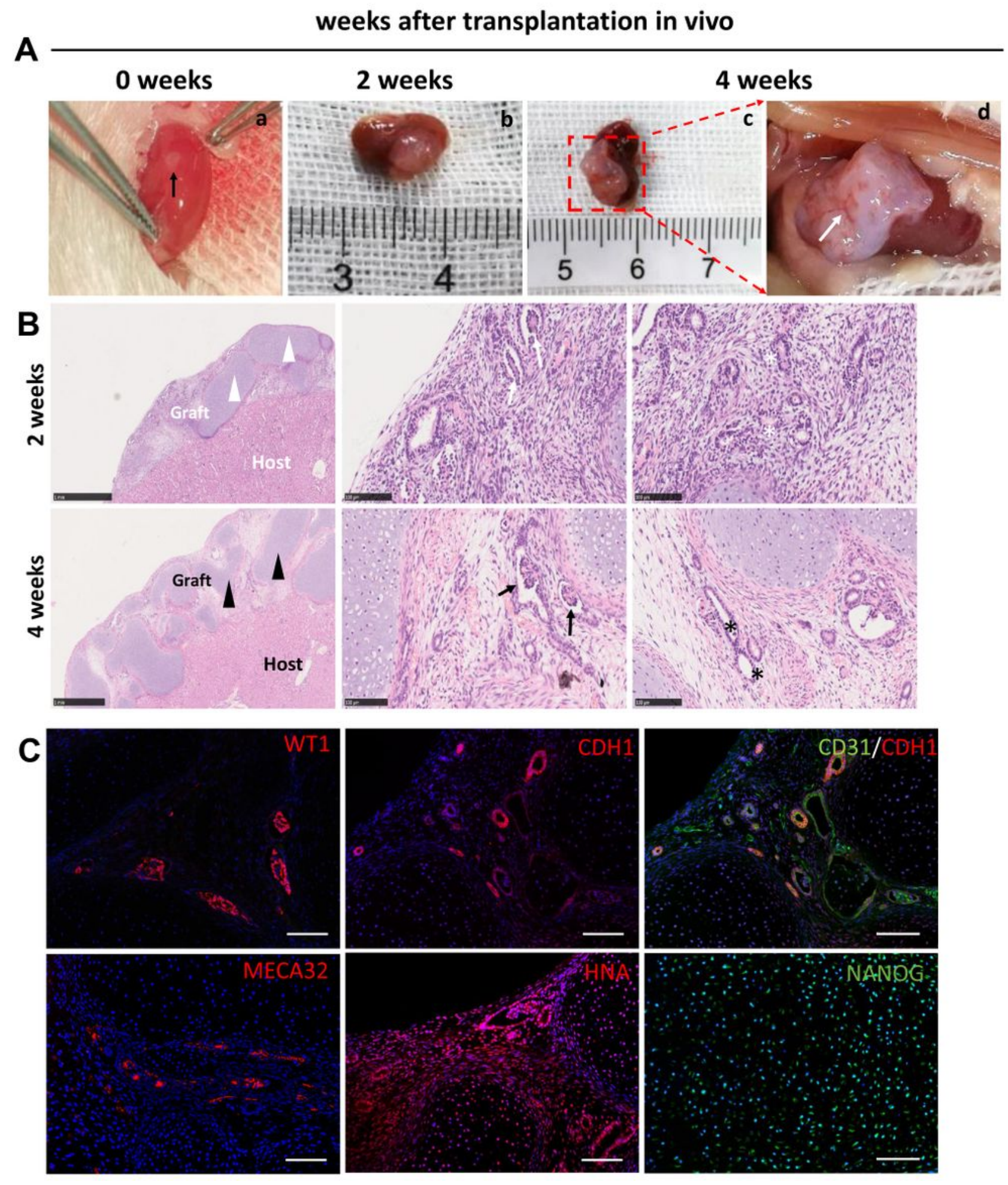

Figure 3

Kidney organoids became vascularized upon transplantation for 2-4 weeks. (A) Transplanted human kidney organoid (black arrows) under the renal capsule of a mouse on the day of transplantation, as well as 2 and 4 weeks later, displaying growth upon vascularization. (B) 2 and 4 weeks after renal transplantation, hematoxylin and eosin staining revealed structures of chondroids (arrowheads), glomeruli (arrows), tubules (asterisks), and erythrocytes. (C) Immunofluorescence assay of renal 
organoids 4 weeks after transplantation. Scale bar, $100 \mu \mathrm{m}$. WT1 expression in podocyte nuclei (top left), $\mathrm{CDH} 1$ immunostaining of large branched tubules (top middle),immunofluorescence of vascular endothelial cell molecular marker CD31 and CDH1 (top right). In addition, mouse endothelial cells (MECA$32+$, bottom left) were observed in association with glomerular structures in human kidney organoids.

Detection of human-derived cell-specific marker human nuclear antigen expression (bottom middle), and NANOG (bottom right) immunostaining of cartilage.
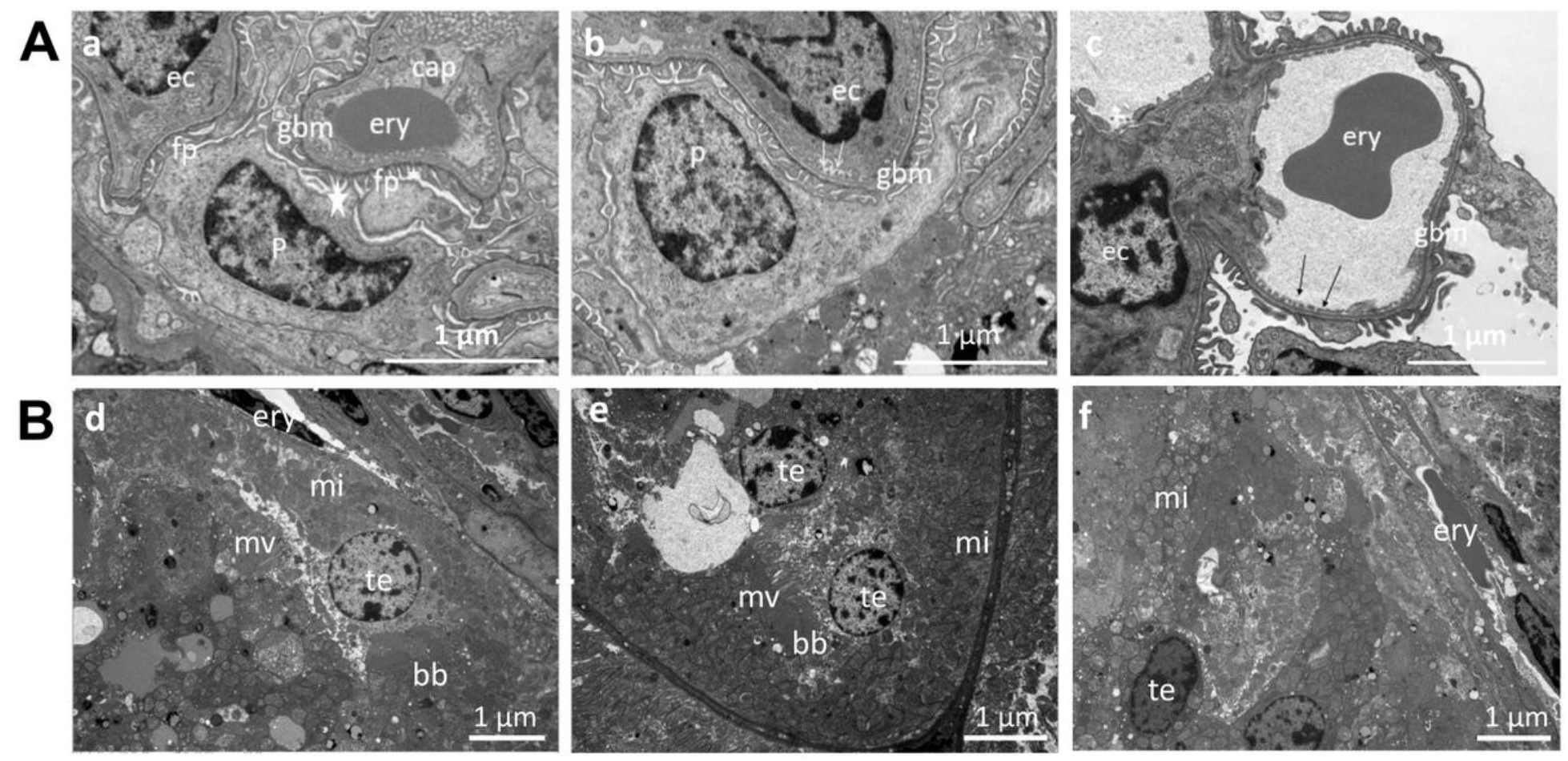

\section{Figure 4}

Ultrastructural assessment of transplanted organoids revealed graft vascularization as well as maturation. (A) Transmission electron micrographs for vascularized glomerular structures after 4 weeks of transplantation. Glomeruli displayed maturation characteristics such as foot processes, glomerular basement membrane processes, and progression toward slit diaphragm formation. p, podocyte; ery, erythrocyte; fp, foot processes; gbm, glomerular basement membrane; ec, endothelial cell; arrows, endothelial fenestrae; cap, asterisk, capillary; developing slit diaphragm. Scale bar, $1 \mu \mathrm{m}$. (B) Transmission electron micrographs of developing tubular structures with peritubular capillaries after 4 weeks of transplantation. Tubular structures became organized and displayed a single layer of epithelial cells connected with tight junctions, as well as an open lumen and tubular polarization, and characteristic mitochondria and microvilli. te, tubular epithelium; ery, erythrocyte; mi, mitochondria; mv, microvilli; bb, brush border. Scale bar, $1 \mu \mathrm{m}$. 

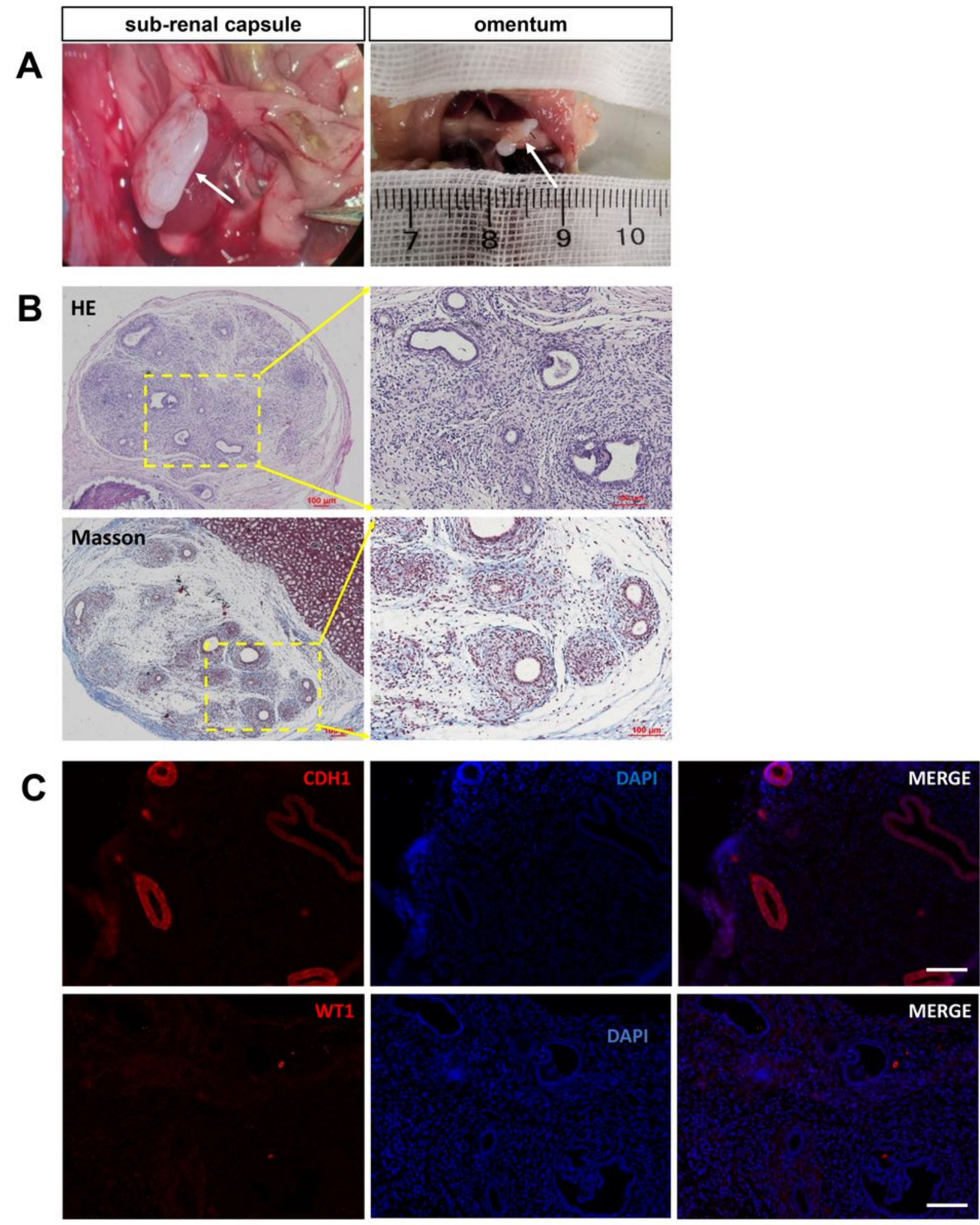

\section{Figure 5}

Histological examination after 12 weeks of renal allograft. (A) The graft (white arrows) volume was further increased after 12 weeks of transplantation. After 12 weeks of subrenal capsuleand para-omental renal transplantation, graft (white arrow) volumes were further increased. (B) Hematoxylin and eosin staining, and Masson staining displayed structures of mesenchymal tissues in grafts after 12 weeks of transplantation. Scale bar, $100 \mu \mathrm{m}$. (C) Immunofluorescence detection of a few distal tubules positive for 
the marker $\mathrm{CDH} 1$ in 12-week grafts. No obvious expression of WT1 was observed in organoids after transplantation for 12 weeks. Scale bar, $100 \mu \mathrm{m}$. 